\title{
Control of the operating temperature of a photovoltaic system using PID
}

\author{
Alex Sander Leocádio Dias ${ }^{1}$, Erlani Silva de Oliveira ${ }^{2}$, José Francisco de Caldas Costa ${ }^{3}$, José Renato da \\ Silva Alencar ${ }^{4}$, Roberto Ferreira de Lima ${ }^{5}$, Paulo Francisco da Silva Ribeiro ${ }^{6}$, Worlen Ferreira Gimack ${ }^{7}$
}

${ }^{1}$ Programa de Pós-Graduação em Engenharia de Processos do Instituto de Tecnologia da Universidade Federal do Pará (ITEC-UFPA). Rua Augusto Correa, 01 - Guamá. CEP: 66075-110. Caixa Postal 479. PABX + 5591 3201-7000. Belém Pará - Brasil.

${ }^{1,6,7}$ Instituto de Tecnologia e Educação Galileo da Amazônia (ITEGAM). Avenida Joaquim Nabuco No 1950. Centro. CEP: 69005-080. Manaus Amazonas - Brasil.

Email:alex.sander06@gmail.com,alex.sander06@gmail.com,josefranciscocaldas@hotmail.com,jrenatosalencar@gmail.com, lima_rf@hotmail.com, paulofranciscosilvaribeiro@gmail.com, worlen_gimack@hotmail.com

Received: August $30^{\text {th }}, 2016$

Accepted: November $11^{\text {th }}, 2016$

Published: December $22^{\text {th }}, 2016$

Copyright (C2016 by authors and Institute of Technology Galileo of Amazon (ITEGAM). This work is licensed under the Creative Commons Attribution International License (CC BY 4.0).

http://creativecommons.org/licenses/by/4.0/

\section{ABSTRACT}

The present article presents a study of the application of the strategy of control of the temperature of operation of a photovoltaic plate using a PID controller. The controller should keep the working temperature of the photovoltaic cells at around 30 degrees Celsius. For the development of this work the PID controller and graphical simulation, environments were used. The objective of this article is the study of the performance of the photocell based on the stabilization of the operating temperature, the simulation was performed through the software MATLAB and Simulink. The results show that it is possible to keep the temperature stable through forced ventilation and the experiment has relevance, since the temperature stabilization method is applicable in energy generation projects, where the ambient temperature is severe.

Keywords: PID, Temperature, Photocell, Matlab.

\section{Controle da Temperatura de operação de um sistema fotovoltaico com utilização de PID}

\section{RESUMO}

O presente artigo apresenta um estudo da aplicação da estratégia de controle da temperatura de operação de uma placa fotovoltaica utilizando um controlador PID. O controlador deve manter a temperatura de trabalho das células fotovoltaicas em torno de 30 graus Celsius. Para o desenvolvimento deste trabalho foi utilizado o controlador PID e ambientes de simulação gráfica. O objetivo deste artigo é o estudo do desempenho da fotocélula com base na estabilização da temperatura de operação, a simulação foi realizada por meio do software MATLAB e Simulink. Os resultados obtidos demonstram que é possível manter a temperatura estável por meio de ventilação forçada e o experimento tem relevância, pois o método de estabilização da temperatura é aplicável em projetos de geração de energia, onde a temperatura ambiente do processo é severa utilizando controladores PID.

Palavras Chaves: PID, Temperatura, Fotocélula, Matlab.

\section{INTRODUÇÃO}

Com a aprovação dos aprimoramentos da RN 482 da ANEEL, a aplicação de geração distribuída, tanto aos níveis de microgeração como em minigeração, ganha notório papel no suprimento de energia elétrica aos consumidores cativos. O presente trabalho a priori se aplica à microgeração fotovoltaica, a qual se apresenta de duas maneiras: microgeração fotovoltaica ligadas à rede (sistema on grid) e microgeração autônomos não conectados à rede (sistemas off grid). No caso da microgeração desconectada da rede, o desempenho do sistema deve ser o melhor possível para o melhor aproveitamento da energia solar disponível na latitude em que o mesmo for instalado, pois uma vez que são poucas horas para produzir e acumular a energia demanda pela instalação, sendo obrigatório um sistema de armazenamento em baterias, fato impactante no custo do sistema inteiro. A celeuma 
sobre a utilização da microgeração fotovoltaica está no fato de apenas uma parte da radiação solar chega até superfície terrestre. A atmosfera bloqueia e reduz a energia solar por meio da reflexão, absorção dos gases e por meio da dispersão em função das partículas em suspenção na atmosfera, sendo grande parte resultado da poluição. Recursos de energia convencionais, que deverão esgotar em um futuro próximo, inevitavelmente, tomar o primeiro lugar no qual a energia de produção global não podem satisfazer a necessidade, devido ao aumento crescente da população e da industrialização.

A diferença entre os aumentos de produção e consumo de energia com cada dia que passa [1]. Adicional decorrentes da produção problemas ambientais eo consumo de fontes de energia convencionais, aumentram o interesse em fontes de energia renováveis em todo o mundo; Especialmente no interesse sistemas fotovoltaicos você e aumentou consideravelmente nos últimos anos. Com o aumento das atividades de pesquisa e desenvolvimento em fotovoltaica, a demanda por sistemas fotovoltaicos cada ano aumentou com uma média de 20-25\% nos últimos 20 anos [2[3].

Todas estas barreiras culminam na diminuição do nível de irradiância que chega à superfície da Terra, a qual estima-se em $1.000 \mathrm{~W} / \mathrm{m}^{2}$ quando o sol está no zênite da latitude, a céu aberto com boas condições climáticas. Ao realizar a somatória da quantidade total da radiação solar incidente durante o período de um ano, é possível se obter a irradiação anual, que é medida em $\mathrm{kWh} / \mathrm{m}^{2}$. Este parâmetro varia de modo significativo de região para região geográfica. $\mathrm{O}$ desempenho do sistema requer um estudo do local, da quantidade de horas de sol pleno e dos componentes do sistema a serem instalados. A temperatura de operação da célula fotovoltaica é o foco da simulação por meio do Matlab [4], além da estratégia de controle por meio de um controlador PID, para verificar se a diminuição da temperatura tem influência no desempenho do sistema.

\section{REVISÃO BIBLIOGRÁFICA}

Na década de 70 do século passado ocorreu uma crise mundial de energia elétrica, demonstrando a fragilidade de toda a civilização frente a uma escassez de combustíveis ditos fósseis, fato que desencadeou a busca por fontes alternativas de energia renováveis, entre as quais a energia solar é abordada neste trabalho. Ainda na década de 90 do mesmo século, houve a concepção dos primeiros sistemas de geração fotovoltaicos, atrelados essencialmente a programas de incentivos governamentais, estes justificados por tratados internacionais como a ECO 92. Ressalte-se que mesmo em países onde o aproveitamento da energia solar é bastante presente em sua matriz energética, citando a Alemanha como exemplo de um país com esta característica, a utilização desta forma de energia ainda encontra muitas barreiras associadas à sua exploração. Entre as barreiras neste trabalho é destacado o custo de produção do $\mathrm{kWh}$ neste tipo de sistema, o qual foi e ainda é um entrave muito grande à expansão e utilização do mesmo com fonte alternativa de energia, conforme demonstra o gráfico da figura 1.

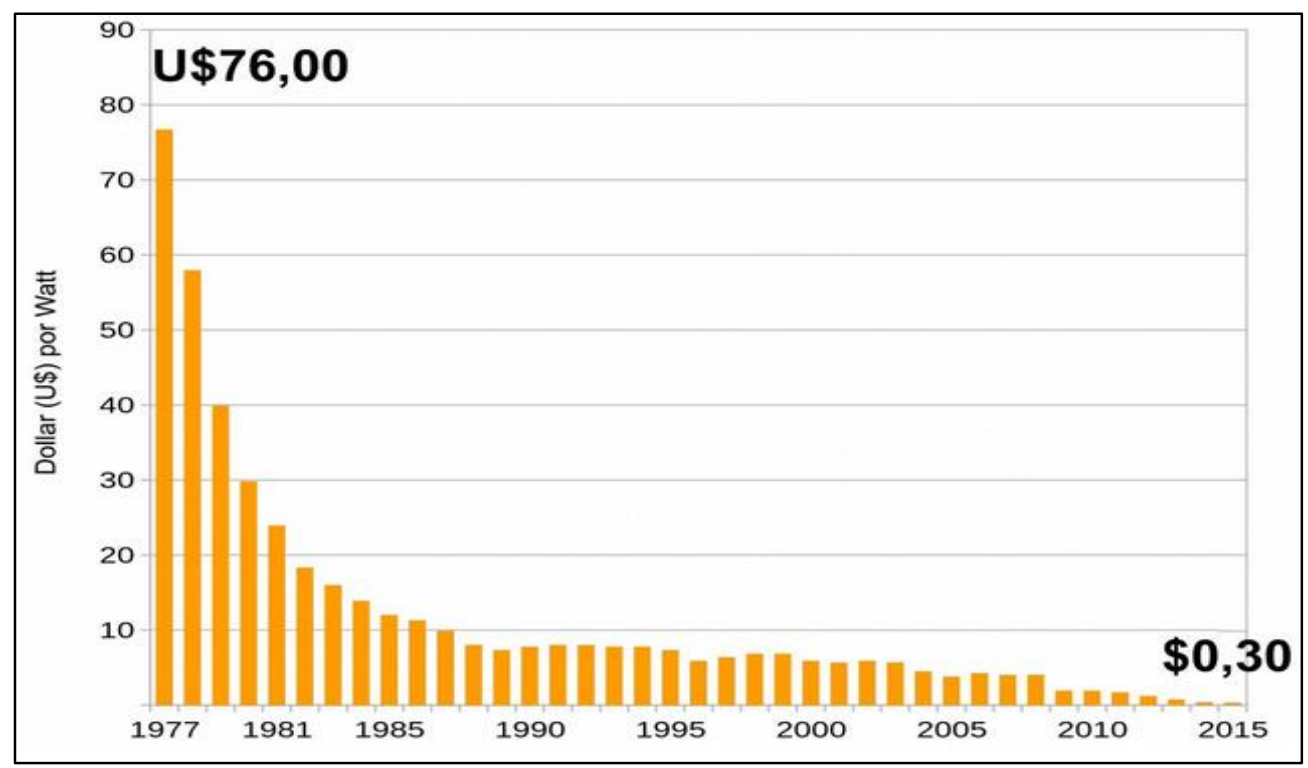

Figura 1. Custo em U\$ (dólar) do kWh em sistemas fotovoltaico Fonte: [5].

Entretanto, quando se trata de geração distribuída e principalmente geração em locais remotos, longe dos centros urbanos e localidades isoladas distantes das redes de distribuição das concessionárias de energia elétrica, a aplicação de sistemas de micro e minigeração toma um papel importante, de cunho social mais do que técnico. No Amazonas a Eletrobrás Distribuição Amazonas, com intuito de atender a universalização da energia elétrica, investiu R \$ 6,6 milhões, através do Programa Federal Luz para Todos, na implementação de miniusinas de geração fotovoltaicas, as quais visavam atender a demanda energética de doze comunidades em seis municípios não supridos pelo sistema de distribuição convencional. A figura 2 demonstra o projeto elétrico simplificado de uma mini usina padrão implementada neste programa. 


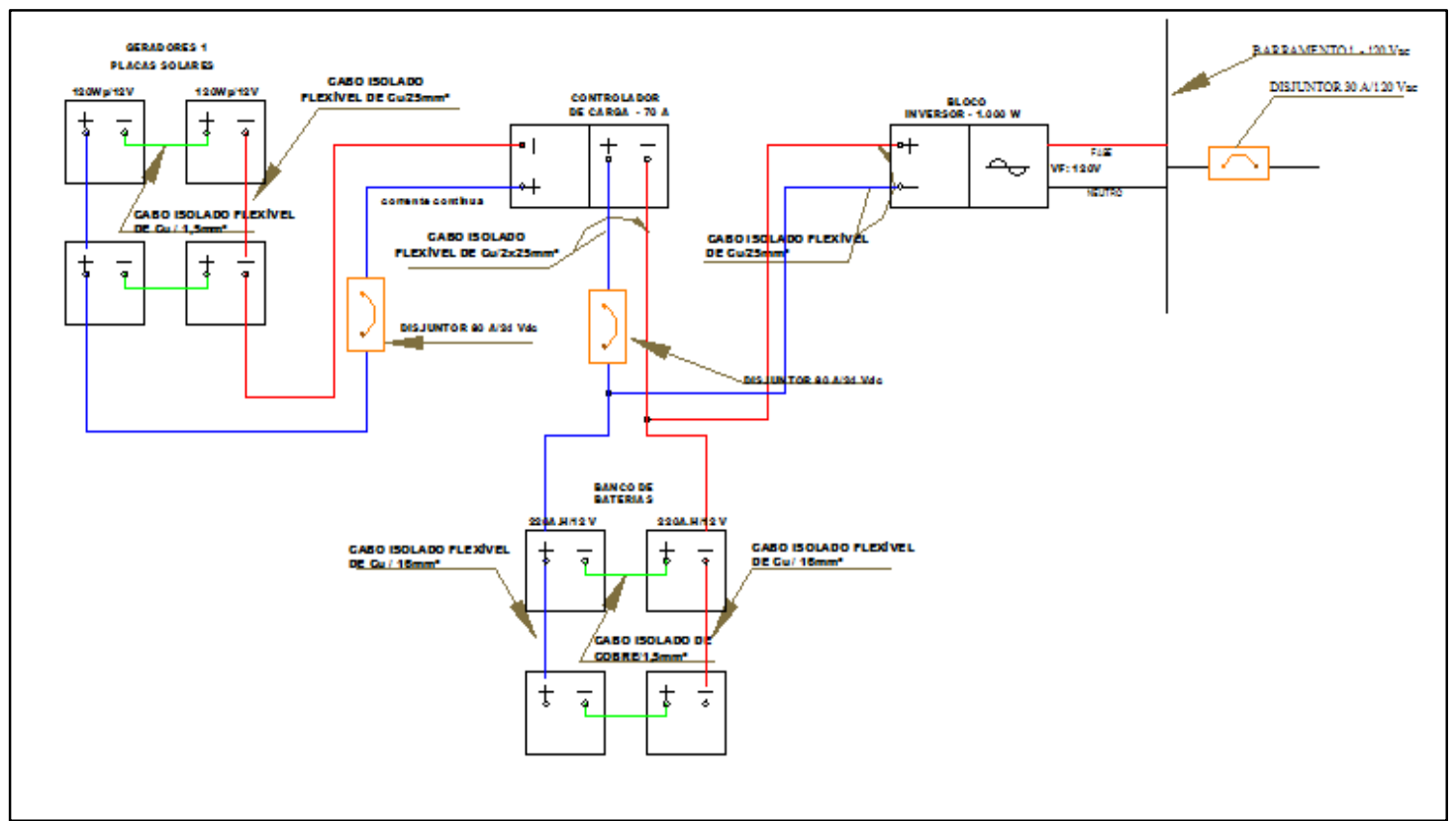

Figura 2. Esquema elétrico simplificado de uma mini usina fotovoltaica.

Fonte: [6].

No presente trabalho é destacada sob o ponto de vista técnico, a queda da potência de saída de um sistema de geração fotovoltaico frente à elevação da temperatura, pois a tecnologia construtiva empregada atualmente usa semicondutores na sua construção elementar (célula fotovoltaica) em configuração de junção PN, sendo esta sensível ao aumento de temperatura. A curva I-V genérica da figura 3, demonstra o efeito nocivo da elevação da temperatura em um sistema fotovoltaico, pois mesmo a elevação da corrente de curto circuito $\left(\mathrm{I}_{\mathrm{sc}}\right)$, a mesma não compensa a queda da tensão de circuito aberto $\left(\mathrm{V}_{\mathrm{oc}}\right)$, como resultante ocorre uma queda na potência fornecida pelo módulo

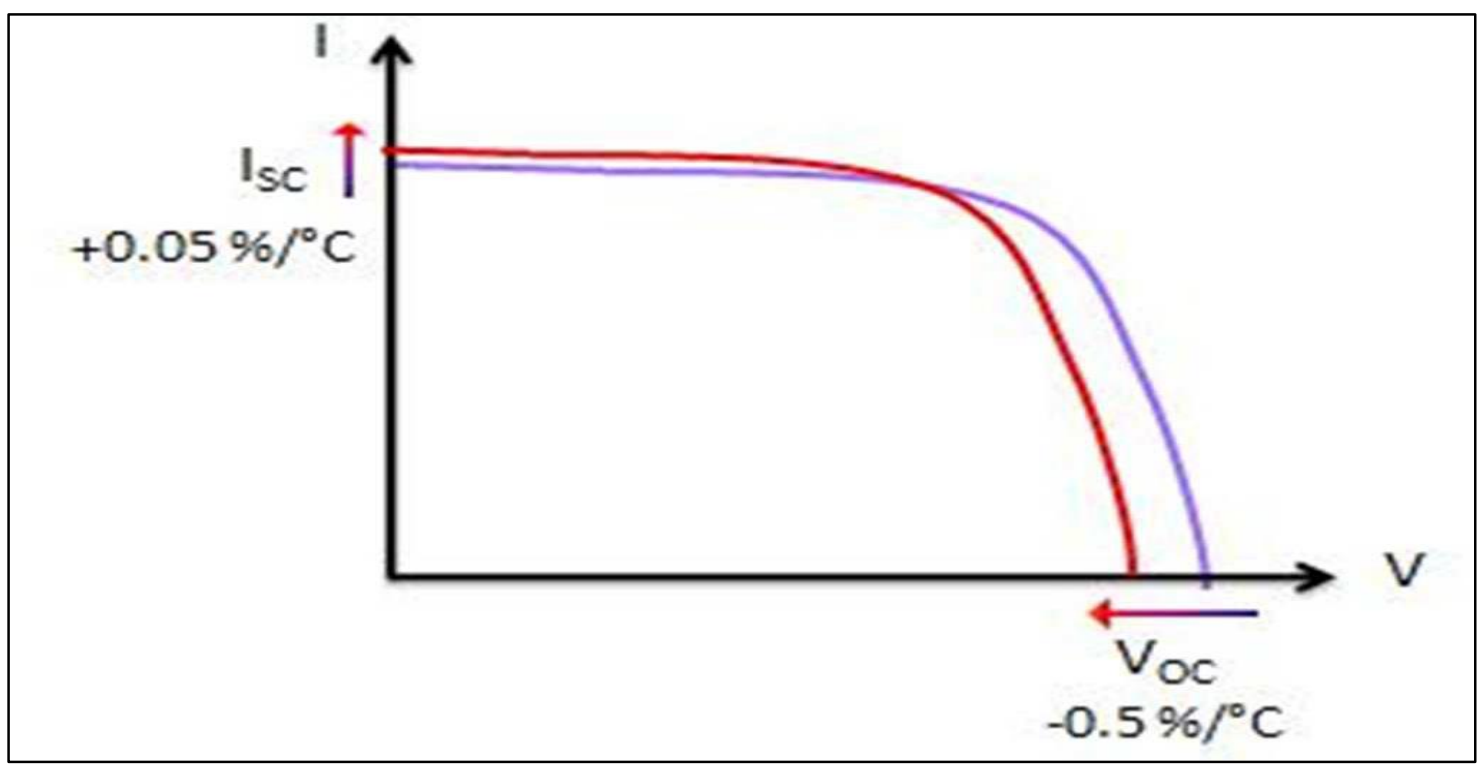

Figura 3. Curva I-V de um gerador fotovoltaico genérico.

Fonte: Autores, (2016).

No modelo de simulação do Matlab, adotado neste trabalho, foi acrescido ao sistema convencional de gerador fotovoltaico uma estratégia de controle do tipo PID [7], buscando estudar o desempenho do sistema com o controle efetivo da temperatura de operação do módulo fotovoltaico, conforme o modelo ilustrado na figura 4 . 


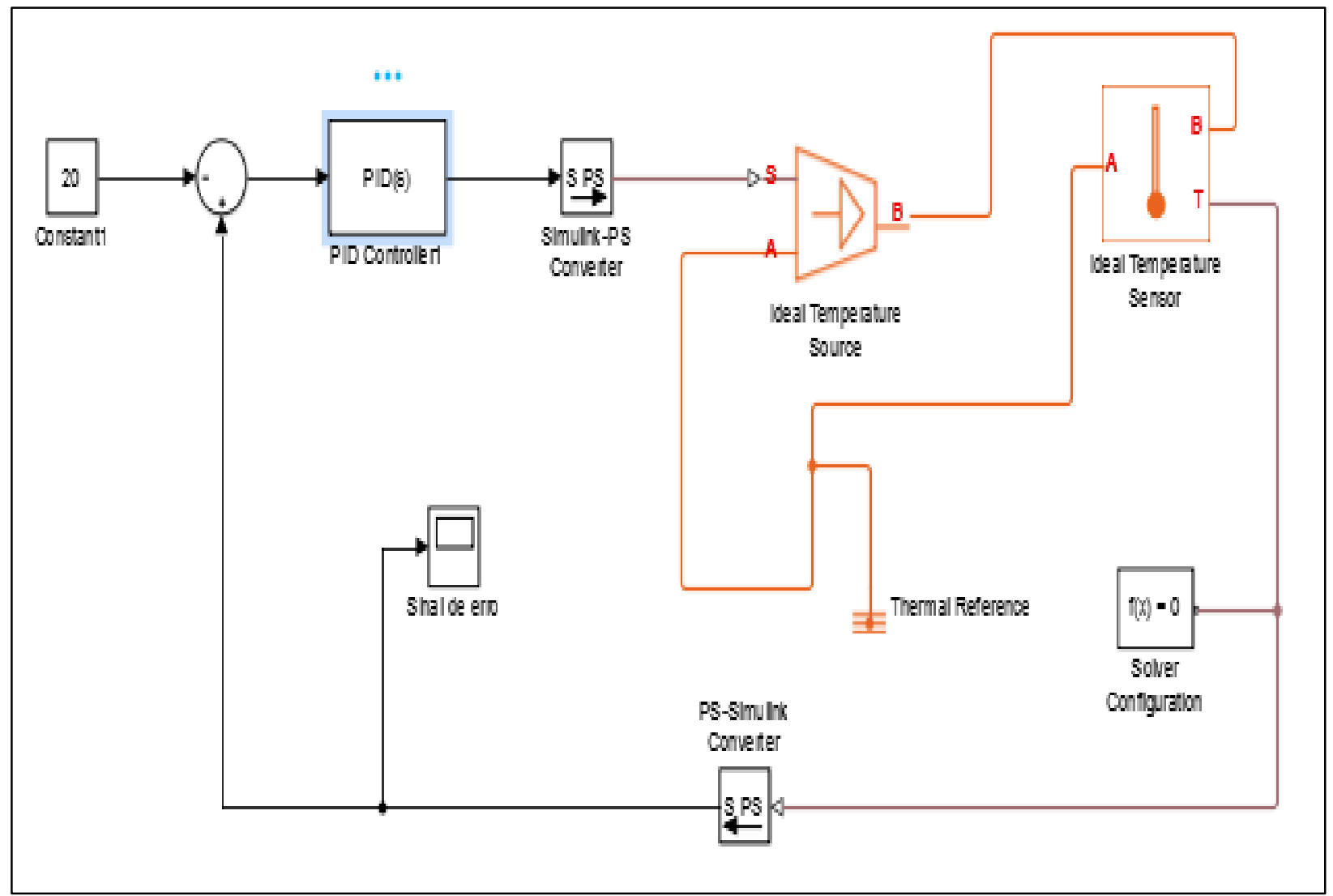

Figura 4. Controlador PID para temperatura.

Fonte: Autores, (2016).

A adoção do controlador PID neste trabalho se deve a robustez e principalmente a versatilidade sobre uma grande gama de aplicações, além de sua simplicidade funcional. Os ganhos ofertados em uma estratégia de controle PID é comumente atrelado aos ajustes dos parâmetros básicos de configuração: $\mathrm{k}_{\mathrm{p}}, \mathrm{T}_{\mathrm{i}}$ e $T_{\text {d. }}$. A equação 1 demonstra a equação de transferência de um controlador PID [8].

$$
G_{c}(s)=k_{1}+\frac{k_{2}}{s}+k_{3} s
$$

Entretanto, boa parte das aplicações utilizam o ajuste dos parâmetros pelo método Zeigler -Nilchos para resposta degrau ou impulso, na tabela 1 é demonstrada a sintonia de um PID pelo método citado.

Tabela 1: Sintonia ao degrau pelo método Z-N.

\begin{tabular}{c|c|c|c}
\hline \multirow{2}{*}{ PID } & $\mathrm{K}_{\mathrm{P}}$ & $\mathrm{T}_{\mathrm{I}}$ & $\mathrm{T}_{\mathrm{D}}$ \\
\cline { 2 - 4 } & $1,2(\mathrm{~T} / \mathrm{L})$ & $2 \mathrm{~L}$ & $0,5 \mathrm{~L}$ \\
\hline
\end{tabular}

Fonte: [8].

$\mathrm{Na}$ simulação descrita neste trabalho o bloco que implementa o algoritmo de controle PID de tempo contínuo e discreto no projeto é demonstrado na figura 5 , o mesmo é dotado de recursos avançados como anti-windup, reset externo e rastreamento de sinal. Além de permitir ajustar os ganhos PID automaticamente usando o botão 'Tune.' (requer Simulink_ControlDesign) [9]. 


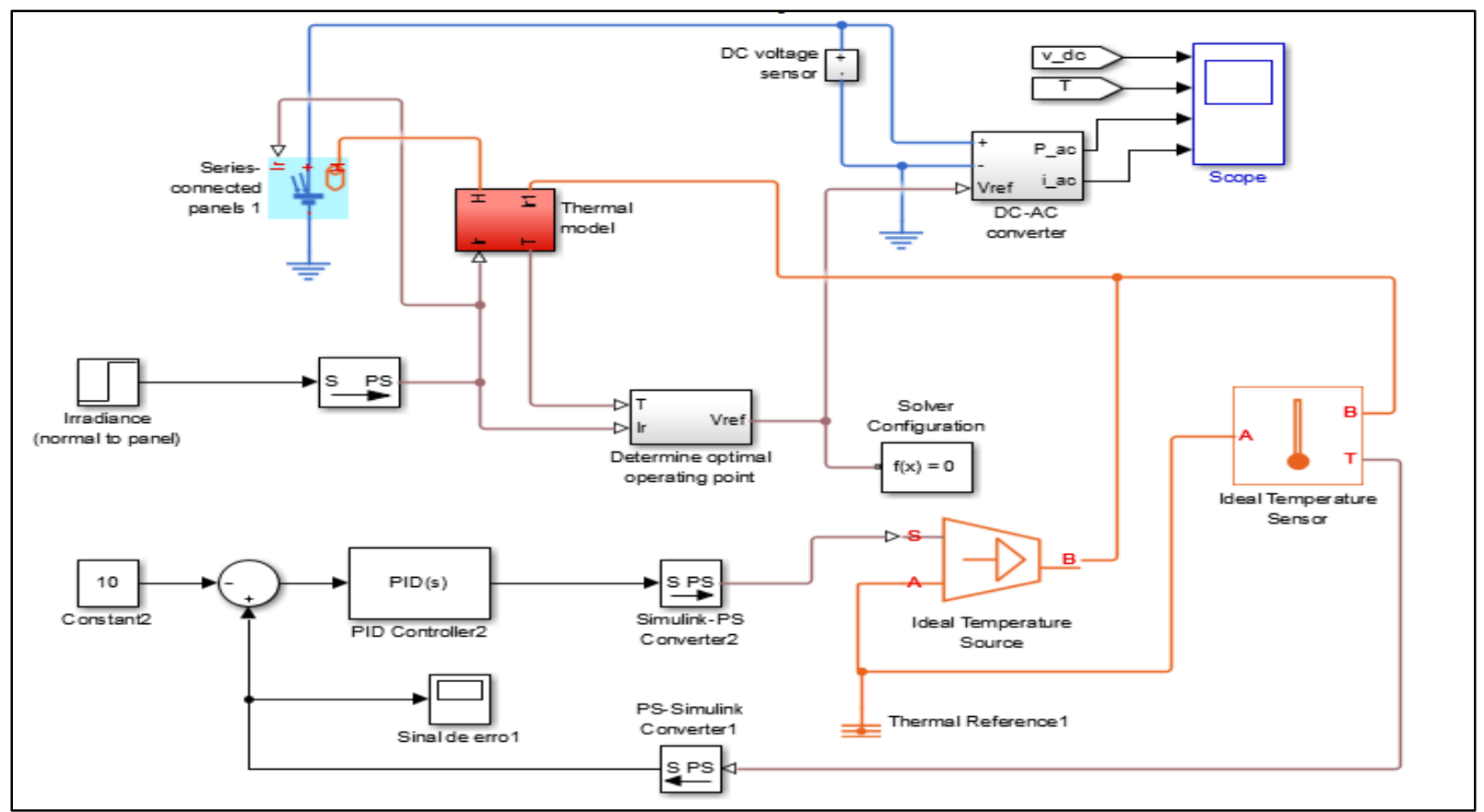

Figura 5. Modelo de Gerador fotovoltaico com controle PID de temperatura.

Fonte: Autores, (2016).

$\mathrm{Na}$ simulação descrita neste trabalho o bloco que implementa o algoritmo de controle PID de tempo contínuo e discreto no projeto é demonstrado na figura 5, o mesmo é dotado de recursos avançados como anti-windup, reset externo e rastreamento de sinal. Além de permitir ajustar os ganhos PID automaticamente usando o botão 'Tune.' (requer Simulink_ControlDesign).

\section{MATERIAIS E MÉTODOS}

Neste tópico é apresentada a metodologia utilizada no desenvolvimento deste trabalho. Para determinação da estratégia de controle foi adotada a de malha fechada demonstrada na figura 6.

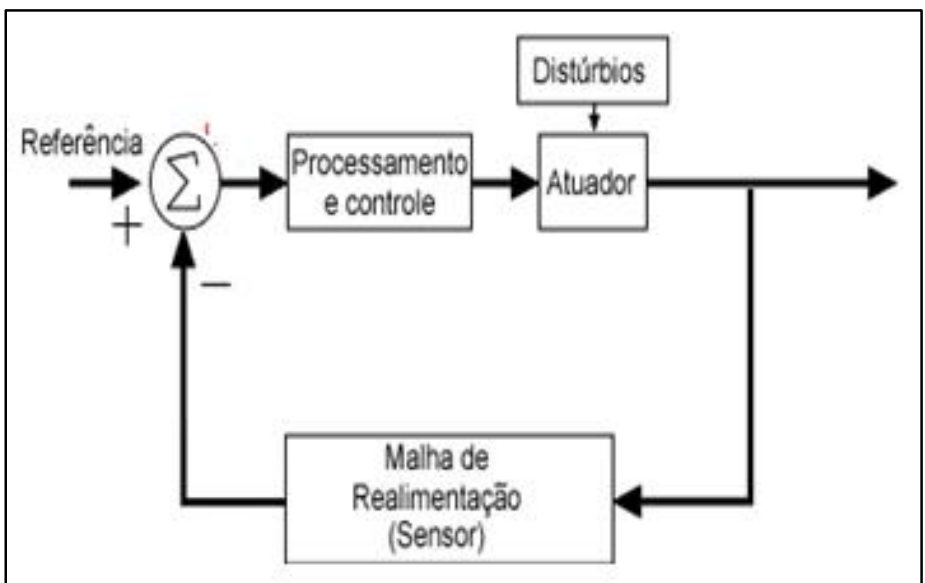

Figura 5. Diagrama de blocos do gerador fotovoltaico com PID Fonte: Autores, (2016).
Para realização deste trabalho foram realizadas modificação de blocos já existentes no software MATLAB, em simulações no módulo SIMULINK. A escolha desta poderosa ferramenta é devido a grande aplicabilidade em desenvolvimento de projetos em várias áreas da engenharia. Por questões práticas as simulações foram realizadas em duas etapas:

a) Utilizando a ferramenta Simulink do MATLAB, foi modificado um exemplo de célula fotovoltaica para atender o projeto, conforme é demonstrado na figura 7 , entretanto, nesta etapa ainda sem a atuação do bloco que simula a ação do controlador PID [10][11];

b) Utilizando a mesma ferramenta, porém com a inserção do bloco que simula a atuação corretiva do controlador PID, conforme o diagrama da figura 4.

\section{RESULTADOS E DISCUSSÕES}

O experimento seguiu as etapas previstas na metodologia, a otimização Ziegler-Nichols foi realizada no MATLAB com a ferramenta Simulink, algumas mudanças foram realizadas para efetuar a interligação entre os modelos já existentes, o que proporcionou a simulação e analise dos resultados obtidos.

A figura 8 ilustra o resultado da simulação de um sistema fotovoltaico [12], no qual é possível notar o aumento da temperatura ambiente e que vai de $29.1^{\circ} \mathrm{C}$ até $38,6^{\circ} \mathrm{C}$, os dados apontam para a perda de rendimento do sistema em função do aumento da temperatura, e como consequência uma diminuição dos valores de tensão $\mathrm{DC}$, de $328 \mathrm{~V}$ para estabilizar em torno de $314 \mathrm{~V}$, corrente AC de $10,065 \mathrm{~A}$ cai para $9,643 \mathrm{~A}$ e a potência AC de $1107 \mathrm{~W}$ cai para $1061 \mathrm{~W}$. 


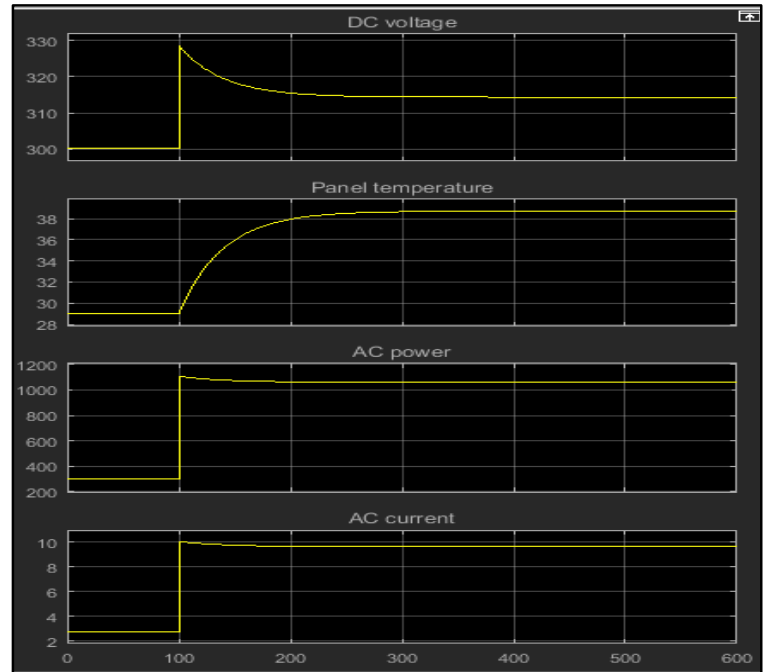

Figura 6. Sinais DC, AC e Temperatura do sistema sem PID. Fonte: Autores, (2016).

Tabela 2. Resultados do experimento sem PID.

\begin{tabular}{c|c|c|c}
\hline Parametro & Inicial & Final & Delta \\
\hline Temperatura & $29.1^{\circ} \mathrm{C}$ & $38,6^{\circ} \mathrm{C}$ & $9,55^{\circ} \mathrm{C}$ \\
\hline Tensão DC & $328 \mathrm{~V}$ & $314 \mathrm{~V}$ & $13,9 \mathrm{~V}$ \\
\hline Potencia AC & $1107 \mathrm{~W}$ & $1061 \mathrm{~W}$ & $46,465 \mathrm{~W}$ \\
\hline Corrente AC & $10,067 \mathrm{~A}$ & $9,643 \mathrm{~A}$ & $0,424 \mathrm{~A}$ \\
\hline
\end{tabular}

Fonte: Autores, (2016).

A figura 9 ilustra o resultado obtido na simulação de um sistema fotovoltaico utilizando um controlador PID, no qual é possível notar a manutenção da temperatura de trabalho, que vai de $20,6^{\circ} \mathrm{C}$ até $33,7^{\circ} \mathrm{C}$, os dados apontam para um rendimento melhor do sistema em função da estabilidade na temperatura, e como consequência uma diminuição das perdas de potência, é possivel verificar que a tensão DC, passa de $341 \mathrm{~V}$ para estabilizar em torno de $322 \mathrm{~V}$, a corrente AC passa de 10,46 A, para estabilizar em 9,64A e a potência AC de $1148 \mathrm{~W}$ estabilizando em $1085 \mathrm{~W}$.

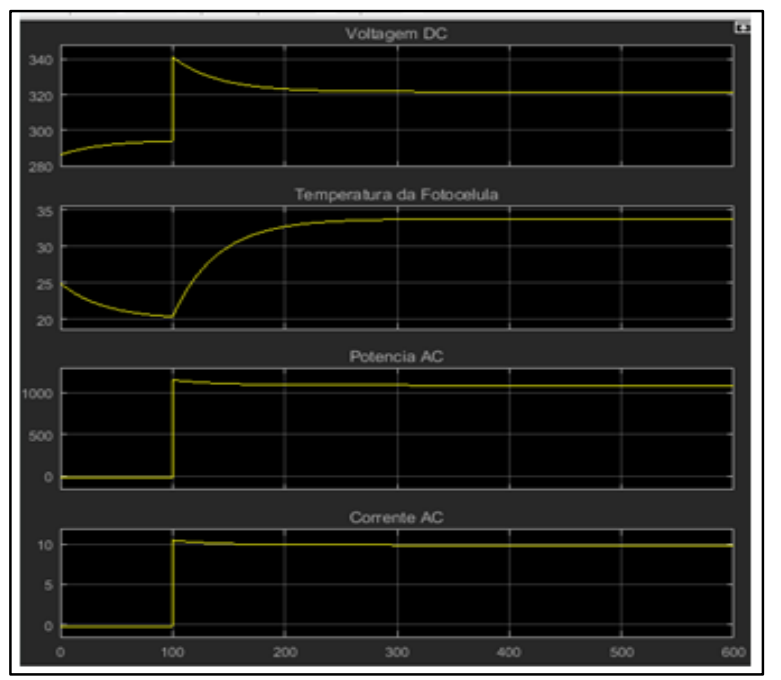

Figura 7. Sinais DC, AC e Temperatura do sistema sem PID.

Fonte: Autores, (2016).
Tabela 3. Resultados com o uso do PID.

\begin{tabular}{c|c|c|c}
\hline Parametro & Inicial & Final & Delta \\
\hline Temperatura & $20.6^{\circ} \mathrm{C}$ & $33,7^{\circ} \mathrm{C}$ & $13,1^{\circ} \mathrm{C}$ \\
\hline Tensão DC & $341 \mathrm{~V}$ & $322 \mathrm{~V}$ & $19,9 \mathrm{~V}$ \\
\hline Potencia AC & $1148 \mathrm{~W}$ & $1085 \mathrm{~W}$ & $62,97 \mathrm{~W}$ \\
\hline Corrente AC & $10,436 \mathrm{~A}$ & $9,863 \mathrm{~A}$ & $0,572 \mathrm{~A}$ \\
\hline \multicolumn{4}{c}{ Fonte: Autores, (2016). }
\end{tabular}

\section{CONCLUSÃO}

O método Ziegler e Nichols é utilizado em muitas aplicações indústrias sendo prático, podendo ser realizado de forma empírica, um especialista em automação e controle de processo, pode desenvolver um projeto com base no Matlab. Ficando a implementação mais rápida e segura. $\mathrm{O}$ experimento mostrou que é possível a implementação de um controle de temperatura de trabalho do sistema fotovoltaico, reduzindo a perda em função do aumento da temperatura.

\section{AGRADECIMENTOS}

Ao Instituto de Tecnologia e Educação Galileo da Amazônia (ITEGAM), e a Universidade Federal do Pará (UFPA).

\section{REFERÊNCIAS}

[1] Kadıoglu, S., \& Tellioglu, Z. (1996). Enerji kaynaklarının kullanımı ve çevreye etkileri. Proceedings of the TMMOB Turkish Energy Symposium (pp. 55-67).

[2] Linares, L., Erickson, R. W., MacAlpine, S., \& Brandemuehl, M. (2009). Improved energy capture in series string photovoltaics via smart distributed power electronics. Proceedings of 24th IEEE Applied Power Electronics Conference and Exposition (pp. 904-910).

[3] Selvaraj, J., \& Rahim, N. A. (2009). Multilevel inverter for grid-connected PV system employing digital PI controller. IEEE Transactions on Industrial Electronics, 56(1), 149-158.

[4] Mathworks Inc. Student Edition of MATLAB Version 2015 for Windows 8 .

\section{[5] http://www.portalsolar.com.br/energia-fotovoltaica.html.}

[6]http://www.brasil.gov.br/infraestrutura/2011/07/eletrobrasinaugura-na-amazonia-quatro-miniusinas-a-luz-solar-em-sistemapre-pago.

[7] Fermino, Fernando. Estudo Comparativo de Métodos de Sintonia de Controladores PID. São Paulo: USP, 2014. Trabalho de conclusão de curso em Engenharia Elétrica. Escola de Engenharia de São Carlos da Universidade de São Paulo, 2014. 
[8] Pinto, Jan Erik Mont Gomery. Aplicação Prática do Método de Sintonia de Controladores PID Utilizando o Método do Relé com Histerese. Rio Grande do Norte: UFRN, 2014. Dissertação (Mestrado em ciências). Centro de Tecnologia - Programa de Pós Graduação em Engenharia Elétrica e de Computação, 2014.

[9] Pinheiro, José Antônio. Desenvolvimento de um Controlador PID para Aplicação em uma Mesa Angular Rotativa. São Paulo: USP, 2009. Dissertação (Mestrado em engenharia mecânica). Escola de Engenharia de São Carlos da Universidade de São Paulo, 2009.

[10] Godoy, Rodrigo Juliani Correa de. Sintonia Ótima de Controladores. São Paulo: USP, 2012. Dissertação (Mestrado em engenharia). Escola Politécnica da Universidade de São Paulo, 2012.

[11] J. Franco M. Amaral, M. A. C. Pacheco, R. Tanscheit, Sintonia de Controladores PID utilizando Algoritmos Genéticos. Rica, Rio de Janeiro, n.08, p. 107-119, Jun/Jul, 2010.

[12] Wolfgang, P. Energia Solar e Fontes Alternativas. São Paulo: Pioneira, 1994. 